\section{Concluding remarks}

The provision of appropriate care for people with severe mental illness in consultation with them, and their informal and formal carers, is a most complex activity. Defining good practice is, however, essential but is an evolving process. The care programme approach provides a description which has general professional support (Social \& Community Planning Research, 1993). Its implementation is progressively focusing the limited resources available on those who need them most.

\section{References}

DHSS (1988) Report of the Committee of Inquiry into the Care and Aftercare of Sharon Campbell. (Chairman: J. Spokes) London: HMSO.
KingDON, D.G. (1992) Interprofessional collaboration in mental health. Journal of Interprofessional Care, 2, 141148.

NHSME (1992) Guidance on the Extension of the Hospital and Community Health Services Elements of the GP Fundholding Scheme from 1 Apri 1993. (EL(92)48). London: NHS Management Executive.

Royal College of PSychiatrists (1991) Good Medical Practice in the Aftercare of Potentially Violent or Vulnerable Patients Discharged from In-patient Psychiatric Treatment. London: Royal College of Psychiatrists.

SOCIAL \& COMMUNITY PLANNING RESEARCH (1993) Factors Influencing the Implementation of the Care Programme Approach. London: HMSO.

Weaver, F. \& PATMORE, C. (1990) United fronts. Health Service Journal, 100, 5223, 1554-1555.

David Kingdon, Senior Medical Officer, Department of Health, Wellington House, 133-135 Waterloo Road, London SE1 8UG

\title{
Who works with adult victims of childhood sexual abuse?
}

\author{
Rob Macpherson and Isam Babiker
}

\begin{abstract}
A survey of mental health professionals in a Bristol NHS trust found that most had experience of therapeutic work with sexually abused patients and over half were currently engaged in such work. Supervision was variable and often considered inadequate. Few responders routinely enquired about historical abuse in the course of psychiatric assesement. The findings indicate a need for agreed strategy involving training, supervision and inter-agency co-operation to deal with this increasingty common problem.
\end{abstract}

\section{Background}

In a recent review, Beitchman et al (1992) concluded that women who reported a history of childhood sexual abuse were more likely than non-abused women to present with a range of sexual and affective disorders, and revictimisation experiences.

Estimates of the prevalence of sexual abuse have varied widely from $6 \%$ to $62 \%$ in different studies (Finkelhor, 1987), and methodological problems have led Markowe (1988) to question the feasibility of such research. There is good evidence (Hobbs \& Wynne, 1987) that diagnosis of sexual abuse in childhood has increased in Britain in recent years. Disclosure of historical abuse in adulthood also appears to be increasing, but this area has received little research attention.

Similarly, there seems to be limited information about the experience of professionals in abuse work. Bisset \& Hunter (1992) found that 73\% of surveyed Grampian and North Tayside general practitioners had seen at least one victim of childhood sexual abuse in the previous two years, many referrals occurring several years after the abuse. A review of the literature revealed no data on the extent of involvement of adult mental health workers in the area. This study aimed to find out which members of the psychiatric multidisciplinary team were engaged in therapy or other work with abused patients, and the nature of supervision for this work.

\section{The study}

A questionnaire was sent to a group of mental health workers attached to six consultant-led 
psychiatric teams, comprising Southmead Trust Mental Health Unit. Ward-based nurses were excluded as it was felt that in-patient treatment was more likely to relate to acute illness episodes than to the longer term issues involved in treating the effects of abuse. Questionnaires were anonymous and covered three main areas in 11 questions: current post and personal details, experience of therapy with abused patients throughout professional career, and current therapeutic work with abused patients. A distinction was drawn between psychological treatment of abused patients aimed specifically at helping such people to work through issues of abuse, and other treatments (which may be psychological) of such patients that did not aim to address these issues.

The questionnaire was sent out on two occasions one month apart so as to maximise compliance.

\section{Findings}

Of 45 questionnaires sent out, 32 were returned. As two professionals were absent through long-term sickness, this represents an overall response rate of $74 \%$. Respondents were three consultants/senior registrars, six clinical assistants, seven senior house officers/registrars, four psychologists/art therapists, ten community psychiatric nurses and two psychiatric social workers. Response rates were poorest (three out of eight) from consultant/senior registrars. There are 21 female and 11 male respondents, who had worked a total of 240 years in mental health, an average of 7.5 years per career.

Six respondents reported no experience of working with abused patients, three of whom were senior house officers in their first six months of training. The total number of abused patients seen for therapeutic work ranged from 0 to 20 per professional, and a total of 173 cases had been seen by the 26 staff with experience of this work. In terms of supervision, six staff reported none, eight had sporadic consultant supervision, seven had peer supervision and four had regular weekly formal supervision. Nursing staff tended to have had peer supervision and trainee psychiatrists reported mainly sporadic consultant supervision.

Of the 32 respondents, 15 (47\%) were currently engaged in therapeutic work of this type, seeing a total of 56 abused patients. They included five clinical assistants, three psychologists/art therapists, five community psychiatric nurses and two senior house officers/registrars. Three clinical assistants and one senior house officer/ registrar reported working without supervision. but the remainder had supervision (form unspecified).
A total of 63 patients were reported who were known to have a history of childhood sexual abuse, but were receiving psychiatric treatment which did not focus on this issue. These patients were reported with similar frequency by the different professions.

Routine enquiry into childhood sexual abuse as part of initial assessment was practised by three respondents when assessing male patients, and by five when assessing female patients, with an additional two staff questioning women routinely 'when factors suggested'.

Generally, there were no barriers to referral or co-working on cases of sexual abuse within the multidisciplinary team, but 12 out of 32 professionals reported that they would not refer to or work with social workers (including one social worker!). Otherwise, referral of such cases was reported between professionals in psychiatry, psychotherapy, psychology, occupational therapy and art therapy.

\section{Comment}

This survey shows that most members of the psychiatric multidisciplinary teams had some experience of therapeutic work with abused patients. On average, professionals had taken on approximately one such case every year of their career, and were currently involved in treating 1.75 cases each. This reflected an extensive service provision considering the long-term nature of this type of work. Reports of supervision were variable, and it could be argued that in only four of 26 cases was it adequate, i.e. regular, formalised and with one supervisor. Of particular concern was that six respondents had carried out such work without supervision, and a further four were engaged in unsupervised therapy with an abused patient.

This type of work is complex and difficult, and often evokes powerful feelings in therapist and patient. An understanding of the transference issues involved in provision of support for the therapist is vital not only for the treatment to be helpful, but also to protect the therapist from personal difficulties within an intense, emotionally charged therapeutic relationship (Babiker, 1993). We believe that regular structured supervision is essential for anyone helping patients with issues concerning sexual abuse. As demand for this type of work increases there will be greater need for all mental health professionals to have formal training in this area.

For a variety of reasons, a substantial number of abused patients were receiving psychiatric treatment which did not focus on issues of abuse. This raises questions about the rationale for offering different treatment regimes. It is also specially relevant in view of data which suggest a 
relationship between childhood sexual abuse and a range of psychiatric disorders (Beitchman et al, 1992).

Only a few respondents asked routinely about childhood sexual abuse as part of initial assessment. This is somewhat surprising in view of the apparent frequency of this problem, and the increasing evidence of its association with psychiatric disorders. Omitting to ask about such experiences tends to overlook their significance and risks colluding with a view that such matters should be 'left alone'.

Most worrying is the finding that the only barrier to interprofessional collaboration was the reported reluctance of some staff to refer patients to social services. The main reason for this appears to be concern about possible conflict between the need for social services departments to ensure child protection and that of the patient to have a supportive and trusting therapeutic relationship which guarantees confidentiality. Although all health professionals should be aware of the commitment to interagency cooperation embodied in Working Together, it appears that many were working with abused patients in relative isolation and blissful ignorance of their obligations under the Children Act 1989.

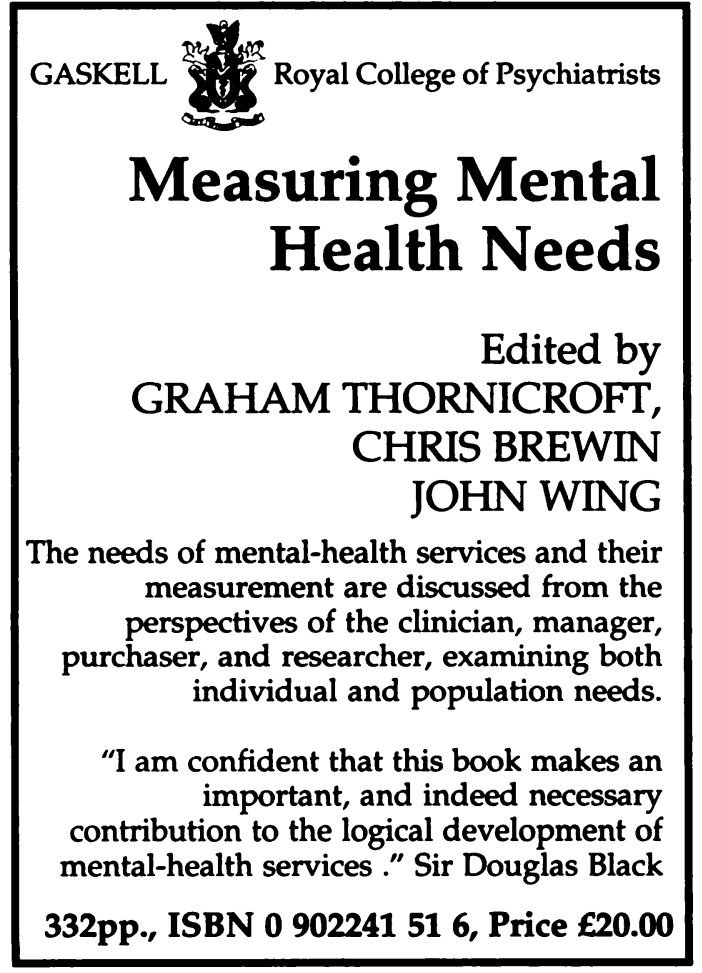

Since this survey, guidelines have been published (Babiker, 1993) and workshops organised to ensure good practice and provide support and supervision for professionals engaged in this type of work.

\section{References}

BABIKER, I.E. (1993) Managing sexual abuse disclosure by adult psychiatric patients - some suggestions. Psychitatric Bulletin, 17, 286-288.

BETTCHMAN, J.H., ZUCKER, K.J., HOOD, J.H., DACOSTA, G.A. et al. (1992) A review of the long-term effects of child sexual abuse. Child Abuse and Neglect, 16, 101-118.

BISSET, A.F. \& HUNTER, D. (1992) Child sexual abuse in general practice in North East Scotland. Health Bulletin. 50, 237-247.

FINKELHOR, D. (1987) The sexual abuse of children: current research reviewed. Psychiatrlc Annals, 17, 233-241.

HOBBS, C.J. \& WYNNE, J.M. (1987) Child sexual abuse - an increasing rate of diagnosis. Lancet, $t, 837-841$.

MARKOWE, H.L.J. (1988) The frequency of childhood sexual abuse in the UK. Health Trends, 20, 2-6.

*Rob Macpherson, Senior Registrar; and Isam Babiker, Consultant Psychiatrist, Mental Health Unit, Southmead Hospital, Westbury on Trym, Bristol BS10 5NB

*Correspondence

\section{Research Methods in Psychiatry 2nd Edition Edited by CHRIS FREEMAN and PETER TYRER}

The popular first edition has been fully revised and updated. Sections on data collection and use of computers have been expanded to incorporate new developments in these fields, and there is additional information on meta-analysis and rating scales. Two new chapters have been added, on writing review papers and research into the history of psychiatry.

“...must become essential reading for those training in psychiatry..." Lancet

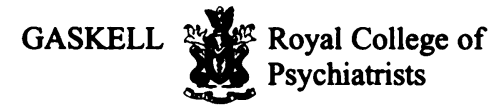

Price £15.00 328pp. ISBN 0902241486 